\title{
Promover o Raciocínio Geométrico em Alunos com Perturbação do Espectro do Autismo através de um Ambiente Digital
}

\author{
Promoting Geometric Reasoning in Students with Autism Spectrum \\ Disorder through a Digital Environment
}

\author{
Maria Isabel Gomes dos Santos* \\ ORCID iD 0000-0002-2655-2239 \\ Ana Maria Reis d'Azevedo Breda ${ }^{* *}$ \\ ORCID iD 0000-0001-7076-707X \\ Ana Margarida Pisco Almeida ${ }^{* * *}$ \\ ORCID iD 0000-0002-7349-457X
}

\begin{abstract}
Resumo
O raciocínio e a construção do sentido espacial são capacidades essenciais em todos os processos de aprendizagem e compreensão matemática de crianças com desenvolvimento típico. Em crianças com Perturbação do Espectro do Autismo (PEA), estas capacidades se tornam ainda muito mais significativas, considerando o papel relevante que desempenham para uma vida independente bem-sucedida. $\mathrm{O}$ uso da tecnologia é referido como uma forma eficaz de trabalhar o conteúdo acadêmico com crianças com PEA, possibilitando a criação de ambientes criativos e construtivos onde se podem desenvolver atividades diferenciadas, significativas e de qualidade. No entanto, o desenvolvimento de aplicações tecnológicas para crianças e jovens com PEA continua a merecer pouca atenção, nomeadamente as que dizem respeito ao desenvolvimento do pensamento geométrico. O objetivo deste artigo é relatar os principais resultados obtidos com crianças com PEA utilizando o ambiente digital Learning Environment on Mathematics for Autistic Children desenvolvido, particularmente, no que se refere à promoção das suas capacidades matemáticas fundamentais em geometria. O "PISA 2015 Mathematics Framework" foi a base teórica utilizada para a recolha dos dados, cuja análise, para além de situar o pensamento geométrico dos alunos participantes entre parcialmente estruturado e estruturado, apontou, também, para o redesenho de algumas das atividades implementadas no ambiente digital, tendo em vista a promoção do pensamento geométrico.
\end{abstract}

Palavras-chave: Tecnologias Digitais. Raciocínio Matemático. Atividades de Aprendizagem Matemática. Capacidades Matemáticas Fundamentais.

\begin{abstract}
Reasoning and sense-making are essential capabilities in all mathematical learning and understanding processes, in children with typical development. In children with Autism Spectrum Disorder (ASD), these capabilities become

\footnotetext{
* Doutora em Multimédia em Educação pela Universidade de Aveiro (UA), Aveiro, Portugal. Endereço para correspondência: Campus Universitário de Santiago, 3810-193, Aveiro, Portugal. E-mail: isabelgomes@ua.pt.

** Doutora em Matemática Pura - especialização em Geometria e Topologia pela Universidade de Coimbra (UC), Coimbra, Portugal. Professora Associada com Agregação da Universidade de Aveiro, Departamento de Matemática, Aveiro, Portugal. Endereço para correspondência: Campus Universitário de Santiago, 3810-193, Aveiro, Portugal. E-mail ambreda@ua.pt.

** Doutora em Ciências e Tecnologias da Comunicação pela Universidade de Aveiro, Aveiro, Portugal. Professora Auxiliar da Universidade de Aveiro, Departamento de Comunicação e Arte, Aveiro, Portugal. Endereço para correspondência: Campus Universitário de Santiago, 3810-193, Aveiro, Portugal. E-mail marga@ua.pt.
} 
much more significant, considering their importance for a successful independent living. The use of technology is considered an effective way to work academic content with children with ASD enabling the creation of creative and constructive environments where we can be develop differentiated, meaningful, and qualitative activities. However, the development of technological applications for children and youth with ASD continues to attract little attention, namely the ones regarding the development of geometric thinking. The purpose of this paper is to report the main results obtained with children with ASD using the digital Learning Environment on Mathematics for Autistic Children developed, particularly regarding the promotion of their fundamental mathematical capabilities in geometry. The "PISA 2015 Mathematics Framework" was the theoretical basis for the data collection. Its analysis allowed to place the geometric thought of the participating students between partially-structured and structured and has also pointed to the redesign of some of the activities implemented in the digital environment, in view of the promotion of geometric thinking.

Keywords: Digital technologies, Mathematical reasoning, Mathematical learning activities, Fundamental mathematical capabilities.

\section{Introdução}

A perturbação do espectro do autismo (PEA) constitui uma perturbação neurodesenvolvimental caraterizada por déficits persistentes nas capacidades de comunicação e interação social, combinados com padrões de comportamento, interesses ou atividades restritos e/ou repetitivos (AMERICAN PSYCHIATRIC ASSOCIATION, 2013). Apresentam igualmente um perfil funcional e cognitivo altamente variável, sendo considerada, pelos profissionais de saúde mental infantil, como uma das problemáticas que causam maior desafio ao nível da intervenção. O perfil de desempenho em Matemática de alunos com PEA é altamente variável (KING; LEMONS; DAVIDSON, 2016).

A pesquisa sobre as capacidades acadêmicas de indivíduos com PEA sublinha a importância de desenvolver estudos que abordem intervenções no processo de ensino e aprendizagem da Matemática (GEVARTER et al., 2016), bem como a utilização de aplicações digitais nesta área, sendo as tecnologias digitais uma área de grande interesse para os indivíduos com esta perturbação (SHANE et al., 2012). No entanto, a proposta de utilização de aplicações digitais para crianças com PEA continua a merecer pouca atenção da comunidade científica, nomeadamente no que diz respeito ao desenvolvimento do raciocínio geométrico.

Os Princípios e Normas para a Matemática Escolar (NCTM, 2007) destacam a necessidade e a importância do ensino da Geometria por ser um domínio em que os alunos podem aprender formas e estruturas geométricas e analisar as suas caraterísticas e relações. A aprendizagem da Geometria é fundamental devendo-se dar especial importância ao desenvolvimento do raciocínio geométrico. O National Council of Teachers of Mathematics (NCTM, 2000) enfatiza que a Geometria constitui uma área natural da Matemática para o desenvolvimento das capacidades de raciocínio e de argumentação dos alunos e sugere a utilização de aplicações interativas para o ensino da Geometria. 
Estudos anteriores sugerem que os computadores e as aplicações digitais podem ser vistos como uma ferramenta auxiliar para aprender e podem ajudar os alunos no que diz respeito à experimentação e à compreensão de conceitos e propriedades geométricas (CHANG et al., 2016), promovendo o trabalho de relações matemáticas no contexto de problemas de palavra (FREIMAN; POLOTSKAIA; SAVARD, 2017). Com as aplicações digitais, os alunos ganham experiência na visualização e manipulação de figuras geométricas e podem desenvolver a capacidade de argumentar sobre essas figuras geométricas (CHANG et al., 2016). Além disso, as tecnologias digitais, ao auxiliarem o processo de ensino e de aprendizagem de alunos com PEA, proporcionam-lhes uma gama de diferentes oportunidades por serem um meio eficaz para apresentar os conteúdos acadêmicos e promover a criação de ambientes criativos e construtivos onde é possível desenvolver atividades diferenciadas e significativas (BURTON et al., 2013).

Como mencionado por English (2016), a avaliação do raciocínio matemático baseado em computador fornece contextos atraentes e interativos para explorar a ampla gama de conceitos matemáticos, capacidades e estratégias que podem potencialmente informar sobre o ensino da Matemática para crianças com PEA.

As atividades de aprendizagem implementadas na primeira versão do protótipo do ambiente digital Learning Environment on Mathematics for Autistic Children (LEMA), um ambiente de aprendizagem digital em desenvolvimento pela Linha Temática Geometrix, para crianças com PEA entre os 6 e os 12 anos, foram concebidas para promover o raciocínio geométrico e projetadas segundo o estudo preliminar efetuado (SANTOS; BREDA; ALMEIDA, 2015) de acordo com os perfis funcionais dos alunos com PEA selecionados para esta pesquisa.

Neste artigo apresentamos as opções metodológicas adotadas e os resultados obtidos na primeira aferição do protótipo do ambiente digital LEMA com 4 utilizadores finais com PEA, tendo essa sido efetuada numa escola de referência de Portugal para alunos com PEA. No final de cada sessão foi realizada uma entrevista semiestruturada com professoras de Educação Especial que acompanharam cada aluno durante a aferição do LEMA sobre a sua eficácia.

\section{Perfil matemático dos alunos com PEA: o caso particular da Geometria}

Existem estudos que revelam que alguns indivíduos com PEA têm capacidades matemáticas superiores à média, sendo considerados matematicamente talentosos. Por outro lado, existem outras pesquisas que indicam que os indivíduos com PEA têm dificuldades em tarefas matemáticas (BAE; CHIANG; HICKSON, 2015; IUCULANO et al., 2014; WEI et al., 
2015). Apesar dos déficits cognitivos que estas crianças podem apresentar, observou-se que a trajetória alterada de desenvolvimento que define esta perturbação do neurodesenvolvimento (por exemplo, desaceleração, estabilização e perda de capacidades) também pode levar a capacidades cognitivas notáveis, podendo as crianças com PEA apresentar "ilhotas" de capacidades em vários domínios, sendo um desses domínios a Matemática (BARON-COHEN, 2015). As crianças com esta perturbação possuem geralmente habilidades médias ou acima da média em operações numéricas (pensamento algorítmico), mas têm dificuldades tanto na compreensão de conceitos matemáticos para aplicações cotidianas, como no raciocínio matemático e na resolução de problemas necessários para resolver problemas de palavras aritméticos (IUCULANO et al., 2014; VAN DOOREN; INGLIS, 2015).

No caso especifico da Geometria e medida, há evidências de que algumas crianças com PEA demonstram uma capacidade de processamento e visualização espacial atípica, que pode ser associada com os pontos fortes e fracos da cognição na visualização espacial (CHABANI, HOMMEL, 2014; YAMADA et al., 2012). Há também relatórios que mostram que estas crianças podem ser muitas vezes mais precisas em áreas como a medição. No entanto, podem ter dificuldades particulares em generalizar a compreensão sobre formas geométricas e em compreender as representações 2D-3D e/ou vice-versa. Elas também podem encontrar dificuldades em aspectos relacionados com as transformações geométricas e dificuldades em compreender a diversidade de formas de representar um objeto matemático (DEPARTMENT FOR EDUCATION AND SKILLS, 2001).

Num estudo de Bae, Chiang e Hickson (2015) foram analisadas as diferenças entre crianças com PEA e crianças com desenvolvimento típico na resolução de problemas de Matemática de cariz não algorítmico. Os autores concluíram, em conformidade com estudos anteriores, que a capacidade de resolução deste tipo de problemas é menor em crianças com PEA, tendo sido indicados como fatores associados a estas dificuldades, a compreensão do enunciado, o vocabulário matemático e o conhecimento matemático do cotidiano.

As instruções para a resolução de problemas matemáticos devem ser explícitas, diretas e práticas para as crianças com PEA, para que elas possam memorizar e aplicar os conhecimentos em diferentes contextos (SU; LAI; RIVERA, 2012). Acresce que as crianças diagnosticadas com a referida perturbação, quando expostas a uma instrução sistemática, conseguem aumentar o seu conhecimento s matemático (SU, 2010).

Da revisão de literatura realizada neste campo temático da Matemática e da PEA podemos aferir que, salvo raras exceções, a Matemática tem sido documentada como uma área relativamente poupada ou uma área que necessita ser reforçada numa grande proporção de 
indivíduos com PEA (CHIANG; LIN, 2007; JONES et al., 2009; WEI et al., 2015).

\subsection{Capacidades matemáticas fundamentais}

A Matemática constitui-se como patrimônio cultural da humanidade e instrumento essencial na comunicação e sistematização lógico-dedutiva do pensamento. É neste contexto que a compreensão matemática se torna fundamental para a transição dos jovens para a vida ativa, uma vez que a Matemática permite resolver problemas, solucionar desafios pessoais, profissionais e sociais, compreender aspectos científicos e deslindar situações encontradas na vida diária (OECD, 2017b).

A Geometria é um meio privilegiado para o desenvolvimento das capacidades de visualização espacial, verbalização, pensamento matemático e intuição, o que contribui para melhorar a capacidade de resolução de problemas, para além de ser um domínio, por excelência, de perceber o mundo físico, interpretar, modificar e antecipar transformações relativamente aos objetos (ABRANTES: SERRAZINA: OLIVEIRA, 1999).

O Programme for International Student Assessment (PISA) em 2015 define sete capacidades fundamentais da Matemática: (1) Comunicação, (2) Matematização, (3) Representação, (4) Raciocínio e Argumento, (5) Elaboração de estratégias para a resolução de problemas (EERP), (6) Usar símbolos, linguagem técnica e formal e operações (ULOS) e (7) Usar ferramentas matemáticas (OECD, 2017a; OECD, 2017b).

Com base no quadro teórico apresentado por PISA 2015 Mathematics Framework (OECD, 2017b). no trabalho realizado por Abrantes, Serrazina e Oliveira (1999), no Currículo Nacional do Ensino Básico (ME-DEB, 2001) e no atual Programa e Metas Curriculares de Matemática para o Ensino Básico (PMEB) de Portugal (BIVAR et al., 2013), procuramos sistematizar no quadro 1 as capacidades fundamentais da Matemática para promover o desenvolvimento da competência matemática do domínio da Geometria, que se constitui o principal quadro teórico de referência para a análise dos dados recolhidos durante a primeira aferição do protótipo LEMA.

\begin{tabular}{|l|l|l|l|}
\hline & $\begin{array}{c}\text { Formular } \\
\text { situações } \\
\text { matemáticas }\end{array}$ & $\begin{array}{c}\text { Empregar conceitos matemáticos, } \\
\text { fatos, procedimentos e raciocínio }\end{array}$ & $\begin{array}{c}\text { Interpretar, aplicar e } \\
\text { avaliar os resultados } \\
\text { matemáticos }\end{array}$ \\
\hline Comunicação & $\begin{array}{l}\text { Ler, descodificar e } \\
\text { dar sentido aos } \\
\text { enunciados, } \\
\text { imagens e /ou } \\
\text { animações; }\end{array}$ & $\begin{array}{l}\text { Articular uma solução, descrever o o } \\
\text { trabalho envolvido na obtenção de uma } \\
\text { solução e/ou resumir e apresentar } \\
\text { resultados matemáticos intermédios }\end{array}$ & $\begin{array}{l}\text { Expressar explicações e } \\
\text { argumentos no contexto do } \\
\text { problema; }\end{array}$ \\
\hline
\end{tabular}




\begin{tabular}{|c|c|c|c|c|c|}
\hline Matematização & $\begin{array}{l}\text { Transformar um } \\
\text { problema definido } \\
\text { no mundo real } \\
\text { num estritamente } \\
\text { matemático }\end{array}$ & \multicolumn{3}{|c|}{$\begin{array}{l}\text { Utilizar uma compreensão do contexto } \\
\text { para guiar ou acelerar o processo de } \\
\text { resolução. }\end{array}$} & $\begin{array}{l}\text { Compreender a extensão e } \\
\text { limites de uma solução } \\
\text { matemática que são uma } \\
\text { consequência do modelo } \\
\text { matemático utilizado. }\end{array}$ \\
\hline Representação & $\begin{array}{l}\text { Criar uma } \\
\text { representação } \\
\text { matemática } \\
\text { (modelo) para a } \\
\text { situação } \\
\text { matemática em } \\
\text { consideração. }\end{array}$ & \multicolumn{3}{|c|}{$\begin{array}{l}\text { Dar sentido, relacionar e utilizar } \\
\text { diversas representações/modelos na } \\
\text { interação com um problema. }\end{array}$} & 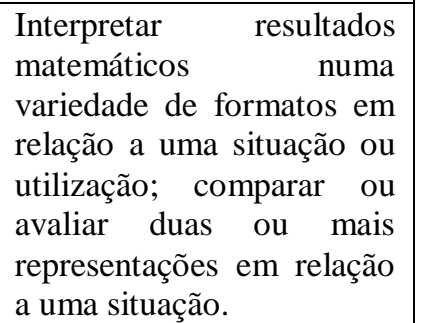 \\
\hline \multirow[t]{12}{*}{$\begin{array}{l}\text { Raciocínio e } \\
\text { Argumentação }\end{array}$} & \multirow[t]{12}{*}{$\begin{array}{l}\text { Explicar, } \\
\text { conjecturar, } \\
\text { defender ou } \\
\text { oferecer uma } \\
\text { justificação para a } \\
\text { identificação, } \\
\text { representação e } \\
\text { resolução de um } \\
\text { problema/situação } \\
\text { matemáticos. }\end{array}$} & \multicolumn{3}{|c|}{$\begin{array}{l}\text { Explicar, defender ou oferecer uma } \\
\text { justificação para os processos e } \\
\text { procedimentos utilizados para } \\
\text { determinar uma solução ou resultado } \\
\text { matemático. Ligar peças de informação } \\
\text { para se chegar a uma solução } \\
\text { matemática. Fazer generalizações ou } \\
\text { criar um argumento multi-passos. } \\
\text { Baseados em: }\end{array}$} & \multirow[t]{9}{*}{$\begin{array}{l}\text { Criar, interpretar, aplicar } \\
\text { avaliar argumentos em defe } \\
\text { de um processo de resoluçà } \\
\text { e/ou prova. }\end{array}$} \\
\hline & & \multirow{8}{*}{\multicolumn{2}{|c|}{ 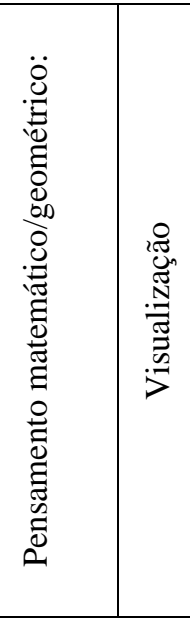 }} & $\begin{array}{l}\text { Coordenação visual- } \\
\text { motora }\end{array}$ & \\
\hline & & & & Percepção figura-fundo & \\
\hline & & & & Constância perceptual & \\
\hline & & & & $\begin{array}{l}\text { Percepção da posição no } \\
\text { espaço }\end{array}$ & \\
\hline & & & & $\begin{array}{l}\text { Percepção das relações } \\
\text { espaciais }\end{array}$ & \\
\hline & & & & Discriminação visual & \\
\hline & & & & Memorização visual & \\
\hline & & & & $\begin{array}{l}\text { Observação } \\
\text { compreensão da ação de } \\
\text { cada uma das } \\
\text { transformações } \\
\text { geométricas }\end{array}$ & \\
\hline & & \multirow{3}{*}{ 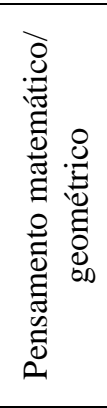 } & \multirow{3}{*}{ 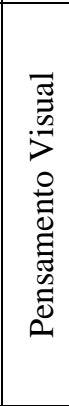 } & $\begin{array}{l}\text { Resultante da percepção } \\
\text { visual }\end{array}$ & \\
\hline & & & & $\begin{array}{ll}\text { Resultante } & \text { da } \\
\text { manipulação mental de } & \text { imagens e construção de } \\
\text { relações entre imagens }\end{array}$ & \\
\hline & & & & $\begin{array}{ll}\text { Resultante } & \text { da } \\
\text { exteriorização } & \text { do } \\
\text { pensamento } & \\
\end{array}$ & \\
\hline $\begin{array}{l}\text { Elaboração de } \\
\text { estratégias } \\
\text { para a } \\
\text { resolução de } \\
\text { problemas }\end{array}$ & $\begin{array}{l}\text { Elaborar } \\
\text { estratégia } \\
\text { resolução } \\
\text { selecionar } \\
\text { estratégias } \\
\text { resolução. }\end{array}$ & \multicolumn{3}{|c|}{$\begin{array}{l}\text { Ativar mecanismos e procedimentos } \\
\text { multi-passo que conduzam a uma } \\
\text { solução matemática, conclusão ou } \\
\text { generalização. }\end{array}$} & $\begin{array}{l}\text { Interpretar, aplicar e validar } \\
\text { a estratégia (selecionada) } \\
\text { no sentido da resolução de } \\
\text { problema. }\end{array}$ \\
\hline \multirow[t]{2}{*}{$\begin{array}{l}\text { Uso de } \\
\text { linguagem e } \\
\text { operações } \\
\text { simbólicas }\end{array}$} & \multirow[t]{2}{*}{$\begin{array}{l}\text { Utilizar } \\
\text { linguagem } \\
\text { matemática } \\
\text { apropriada }\end{array}$} & \multicolumn{3}{|c|}{$\begin{array}{l}\text { Compreender conceitos geométricos } \\
\text { como amplitude, comprimento, área, } \\
\text { volume, unidades de mediada, sistemas } \\
\text { de numeração, etc. baseados em } \\
\text { definições, regras e propriedades. }\end{array}$} & $\begin{array}{l}\text { Compreender a relação } \\
\text { entre o contexto do } \\
\text { problema e a representação } \\
\text { matemática da solução. } \\
\text { Utilizar esta compreensão }\end{array}$ \\
\hline & & \multicolumn{3}{|c|}{$\begin{array}{l}\text { Aplicação de fórmulas } \\
\text { procedimentos algébricos. }\end{array}$} & $\begin{array}{l}\text { como apoio à resolução do } \\
\text { problema e sua validação. }\end{array}$ \\
\hline
\end{tabular}




\begin{tabular}{|c|c|c|c|}
\hline $\begin{array}{l}\text { Uso de } \\
\text { ferramentas } \\
\text { matemáticas }\end{array}$ & $\begin{array}{l}\text { Utilizar } \\
\text { ferramentas } \\
\text { matemáticas } \\
\text { (ambientes } \\
\text { digitais, softwares } \\
\text { de geometria } \\
\text { dinâmica, } \\
\text { máquinas } \\
\text { calcular, ...) para } \\
\text { modelar, } \\
\text { compreender e/ou } \\
\text { interpretar } \\
\text { situações } \\
\text { matemáticas, bem } \\
\text { como para apoiar } \\
\text { atividades de cariz } \\
\text { algorítmico. }\end{array}$ & $\begin{array}{l}\text { Conhecer e ser capaz de fazer uso } \\
\text { adequado de ferramentas matemáticas } \\
\text { para apoio de atividades matemáticas. }\end{array}$ & $\begin{array}{l}\text { Utilizar e } r \text { aplicar } \\
\text { ferramentas matemáticas } \\
\text { adequadas à resolução de } \\
\text { um dado problema, } \\
\text { analisando a razoabilidade } \\
\text { da solução encontrada. }\end{array}$ \\
\hline
\end{tabular}

Quadro 1 - Quadro teórico proposto referente às capacidades fundamentais da matemática (coluna mais à esquerda) no domínio da geometria em relação aos processos matemáticos (top da linha horizontal) (OECD, 2017b).

Fonte: Elaborado pelos autores em 2016.

\section{Desenho do LEMA}

Como mencionado anteriormente, o LEMA foi concebido para promover o raciocínio geométrico por crianças diagnosticadas com PEA e projetado a partir de um estudo preliminar (SANTOS; BREDA; ALMEIDA, 2015), de acordo com os perfis funcionais dos 4 alunos com PEA selecionados para esta investigação.

Nas sessões exploratórias incluídas no estudo preliminar, foram realizadas atividades matemáticas, desenhadas de acordo com os Programas Educativos Individuais (PEI) ${ }^{1}$ de cada aluno utilizando o GeoGebra (Software de Matemática Dinâmica). O estudo preliminar mostrou que os sete participantes apresentaram o raciocínio matemático pouco estruturado ou nulo, revelando um baixo nível de percepção visual (2D-3D-2D). Obtivemos também evidências que a resolução de problemas que envolvam maior capacidade de abstração e justificação é uma área que carece ser trabalhada com estes alunos, especialmente no domínio da Geometria e Medida (SANTOS; BREDA; ALMEIDA, 2015). Estes resultados reforçaram a nossa intenção de desenvolver um protótipo de ambiente digital para alunos com PEA, especificamente orientado para promover o desenvolvimento de capacidades matemáticas no domínio da

\footnotetext{
${ }^{1}$ O Programa Educativo Individual era o documento formal em 2015 que descrevia o perfil de funcionalidade por referência à Classificação Internacional da Funcionalidade: Crianças e Jovens (CIF-CJ) do aluno e estabelece as respostas educativas específicas requeridas para cada aluno, baseadas na observação e avaliação de sala de aula e nas informações complementares disponibilizadas pelos participantes no processo. Este documento responsabilizava a escola e os encarregados de educação pela implementação de medidas educativas que promovam a aprendizagem e a participação dos alunos com necessidades educativas especiais de carácter permanente.
} 
Geometria e Medida. Além disso, estes resultados deram-nos orientações para identificar as principais caraterísticas do protótipo a ser desenvolvido, ou seja, o LEMA.

O processo de especificação e desenho das atividades matemáticas a implementar no protótipo foi realizado com base na revisão de literatura, nos dados recolhidos no estudo preliminar realizado e de acordo com os perfis funcionais dos quatro estudos de caso selecionados para esta fase da investigação (SANTOS; BREDA; ALMEIDA, 2015) e no cumprimento dos requisitos descritos em trabalhos anteriores (SANTOS; BREDA; ALMEIDA, 2017). Na concepção das atividades matemáticas também tivemos em consideração o Programa e Metas Curriculares Matemática para o Ensino Básico (BIVAR et al., 2013) em Portugal. Convém ainda mencionar que as atividades concebidas para os 4 alunos são semelhantes às atividades realizadas no estudo preliminar, contemplando ajustamentos ao perfil dos alunos envolvidos.

Importa referir também que no ensino da Matemática, no que diz respeito ao desenvolvimento do raciocínio matemático, as atividades propostas aos alunos constituem um dos aspectos centrais para o sucesso destes, sendo relevante o tipo de atividade em que os alunos se envolvem, o modo como se envolvem e as interações que podem surgir em torno das atividades (MATA-PEREIRA; PONTE, 2018).

O LEMA incorpora 32 atividades baseadas num conjunto de funções que permitem a manipulação direta de itens visuais (cf. Figura 1). Para além da ilustração simples e cuidadosa dos layouts de cada atividade, foram também incorporados feedbacks de reforço automáticos com animações visuais e sonoras e, em algumas atividades, incorporaram-se feedbacks tutoriais. Os feedbacks são estratégias-chave para crianças com PEA para melhorar a sua precisão matemática de resolução de problemas, a explicação dos conceitos matemáticos e completar problemas mais complexos de forma autónoma (DELMOLINO et al., 2013).

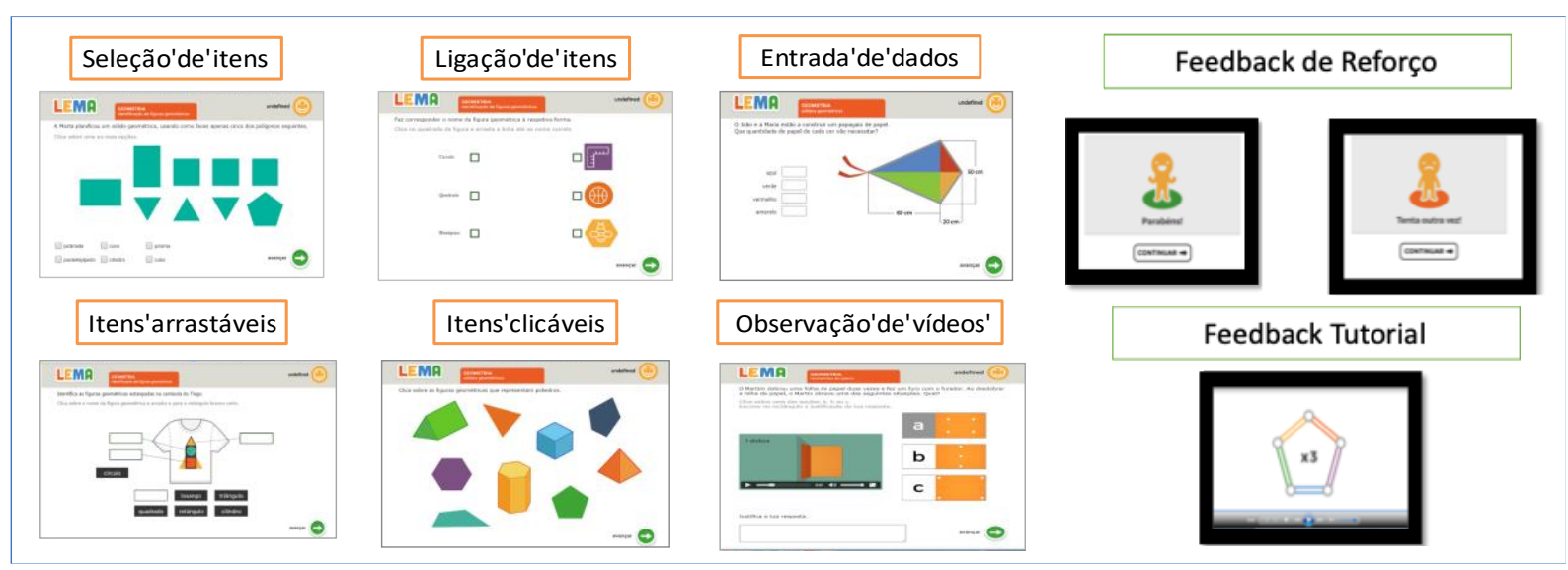

Figura 1 - Elementos incorporados nas atividades do LEMA Fonte: SANTOS (2018) 
Para cada atividade implementada no LEMA, foram identificadas as capacidades matemáticas fundamentais envolvidas, tendo em consideração o referencial teórico apresentado no quadro 1 e os objetivos a atingir (cf. Quadro 2).

\begin{tabular}{|c|c|}
\hline Atividades & Objetivos \\
\hline AT 1 & Associar a planificação do cubo com o cubo correspondente; \\
\hline AT2 & Identificar figuras geométricas em várias posições; \\
\hline AT3 & Identificar figuras geométricas em figuras da vida cotidiana; \\
\hline AT4 & Identificar figuras geométricas em figuras da vida cotidiana; \\
\hline AT5 & Identificar e contar todos os triângulos inscritos num triângulo; \\
\hline AT 6 & Identificar e contar todos os retângulos inscritos num retângulo; \\
\hline AT 7 & $\begin{array}{l}\text { Identificar sólidos geométricos representantes de contextos do mundo real, através da sua } \\
\text { representação no plano; }\end{array}$ \\
\hline AT 8 & Associar o cubo com as respectivas planificações; \\
\hline AT 9 & Associar sólidos geométricos com as respectivas planificações; \\
\hline AT 10 & Identificar vértices, arestas e faces de poliedros; \\
\hline AT 11 & $\begin{array}{l}\text { Reconhecer prismas e pirâmides por meio das suas propriedades combinatórias; Resolver } \\
\text { problemas envolvendo sólidos geométricos e as respectivas planificacões; }\end{array}$ \\
\hline AT 12 & Reconhecer propriedades geométricas dos sólidos através das suas relações espaciais; \\
\hline AT 13 & $\begin{array}{l}\text { Reconhecer propriedades geométricas dos sólidos através das suas relações espaciais, explicando } \\
\text { o raciocínio matemático que conduziu à conclusão; }\end{array}$ \\
\hline AT 14 & Distinguir entre polígonos e poliedros; \\
\hline AT 15 & Completar figuras planas de modo que fiquem simétricas relativamente a um eixo; \\
\hline AT 16 & Realizar processos de pensamento visual explicando o raciocínio matemático desses processos; \\
\hline AT 17 & Realizar processos de pensamento visual explicando o raciocínio matemático desses processos; \\
\hline AT 18 & Identificar isometrias do plano: rotação, translação e reflexão; \\
\hline AT 19 & Resolver problemas envolvendo as propriedades das isometrias no plano; \\
\hline AT 20 & Resolver problemas envolvendo a composição de reflexões em eixos paralelos; \\
\hline AT 21 & Identificar pares de figuras congruentes; \\
\hline AT 22 & Resolver problemas envolvendo figuras com simetrias de rotação e de reflexão axial \\
\hline AT 23 & Decomposição e composição de figuras no plano; \\
\hline AT 24 & Determinar a medida de área de figuras planas, fixada uma determinada unidade de área. \\
\hline AT 25 & Comparar áreas de figuras utilizando uma grelha quadrangular; \\
\hline AT 26 & $\begin{array}{l}\text { Determinar a área de um retângulo/triângulo em unidades quadradas multiplicando o } \\
\text { comprimento pela largura/metade deste, envolvendo conversão de unidades; }\end{array}$ \\
\hline AT 27 & $\begin{array}{l}\text { Resolver problemas envolvendo o cálculo de áreas de retângulos e triângulos por decomposição de } \\
\text { uma dada figura; }\end{array}$ \\
\hline AT 28 & Resolver problemas envolvendo o cálculo de áreas de figuras planas. \\
\hline AT 29 & Resolver problemas envolvendo o perímetro de um polígono; \\
\hline AT 30 & Resolver problemas envolvendo o perímetro de um polígono; \\
\hline AT 31 & $\begin{array}{l}\text { Resolver problemas envolvendo o perímetro de um polígono, explicando o raciocínio matemático } \\
\text { por detrás da conclusão; }\end{array}$ \\
\hline AT 32 & $\begin{array}{l}\text { Resolver problemas envolvendo o perímetro de um polígono, explicando o raciocínio matemático } \\
\text { da conclusão; }\end{array}$ \\
\hline
\end{tabular}

Quadro 2 - Atividades incorporadas no LEMA Fonte: SANTOS (2018)

\section{Metodologia}

O processo de seleção dos participantes para o estudo obedeceu aos seguintes critérios: (1) crianças diagnosticadas com PEA; (2) idades compreendidas entre 6-12 anos; (3) alunos sem outra patologia associada e (4) alunos com a autorização confirmada para a participação 
no estudo, por parte dos encarregados de educação. A identidade e a integridade de todos os participantes foram preservadas. Para elaborar a nossa proposta, foram selecionados 4 dos 7 alunos que participaram no estudo preliminar. Todos os alunos são do sexo masculino, com idades compreendidas entre os 8 e os 12 anos (SANTOS; BREDA; ALMEIDA, 2015).

A fim de avaliar a adequação científico-pedagógica das atividades prototipadas com enfoque no desenvolvimento do raciocínio matemático e no nível do pensamento geométrico dos alunos, foram selecionadas, do total das 32 atividades disponíveis no LEMA, 11 atividades para cada aluno, tendo em conta o seu perfil funcional e as capacidades subjacentes ao raciocínio matemático a serem desenvolvidas, perfazendo um total de 44 tarefas realizadas.

Para cada aluno, após a escolha das atividades matemáticas, construiu-se um grafo regulando a sequência como as atividades seriam lançadas no ambiente digital LEMA. Na construção deste grafo foram consideradas quer as capacidades matemáticas de cada um deles, quer o nível de dificuldade das atividades. Tal como refere Mata-Pereira e Ponte (2018) a articulação entre atividades com diferentes níveis de exigência e de desafio é essencial para o desenvolvimento do raciocínio matemático dos alunos.

O processo de avaliação foi dividido em duas fases: a primeira consistiu na avaliação do nível de raciocínio geométrico, tendo em consideração o quadro teórico de referência resumido no Quadro 1; e a segunda fase na averiguação da adequação científica-pedagógica das atividades implementadas e compreensão da forma como o LEMA pode apoiar os alunos com PEA no processo de ensino e de aprendizagem da Matemática, em particular no tópico da Geometria. Este entendimento foi sustentado na análise das entrevistas realizadas com os professores que acompanharam os alunos durante as sessões. Todas as entrevistas foram gravadas e as transcrições foram codificadas e analisadas com o WebQda (Web Qualitative Data Analysis).

\section{Procedimentos}

A fim de avaliar a adequação científica e pedagógica do conjunto de atividades específicas implementadas no ambiente digital LEMA e o nível do pensamento geométrico dos 4 utilizadores finais selecionados, duas ferramentas metodológicas foram criadas: (1) grelhas de observação para as sessões regulares e (2) guiões para entrevistas aos professores que acompanharam os alunos durante as sessões.

Para a avaliação da capacidade do raciocínio matemático em Geometria dos alunos participantes, foram definidas três categorias: estruturado; parcialmente estruturado; e nulo. 
Para a capacidade de argumentação também foram definidas três categorias: total; parcial; e nula. Estas categorias foram atribuídas de acordo com o número de tentativas que cada utilizador necessitou para dar uma resposta correta e o nível da justificação da resposta sempre que solicitada, ou seja, as categorias foram atribuídas considerando o desempenho dos 4 utilizadores finais na interação com cada atividade implementada no LEMA.

Em seguida apresentamos os critérios utilizados na definição da categorização do raciocínio matemático e argumentação:

1. Se o utilizador dá uma resposta correta na primeira tentativa de resolução de uma dada atividade e nenhuma justificação é requerida, então determinamos que o utilizador tem um raciocínio matemático estruturado, a menos que ocorra a situação descrita no ponto 5;

2. Se o utilizador dá uma resposta correta na primeira tentativa de resolução de uma dada atividade e a justificação é requerida, e o utilizador dá uma justificação completa/incompleta/inexata/nenhuma justificação do seu raciocínio, então determinamos que o utilizador tem um raciocínio matemático estruturado com argumento totall parcial/nulo/nulo respectivamente, a menos que ocorra a situação descrita no ponto 5;

3. Se o utilizador dá uma resposta correta na segunda ou terceira tentativa de resolução de uma dada atividade e nenhuma justificação é requerida, então determinamos que o utilizador tem um raciocínio matemático parcialmente estruturado, a menos que ocorra a situação descrita no ponto 5;

4. Se o utilizador dá uma resposta correta na segunda ou terceira tentativa de resolução de uma dada atividade e a justificação é requerida, e o utilizador dá uma justificação completa/incompleta/inexata/nenhuma justificação do seu raciocínio, então determinamos que o utilizador tem um raciocínio matemático parcialmente estruturado com argumento total/parcial/nulo/nulo respectivamente, a menos que ocorra a situação descrita no ponto 5;

5. Se o utilizador, na terceira tentativa de resolução de uma dada atividade, dá uma resposta incorreta, avança para a próxima atividade sem responder à atividade proposta ou há indicações (notas de registro da observação) que a resposta correta, na primeira, segunda ou terceira tentativa de resolução da atividade, foi dada ao acaso ou utilizando a estratégia de tentativa e erro, então determinamos que o utilizador tem um raciocínio matemático nulo.

\section{Resultados}

Para a avaliação da capacidade de raciocínio matemático dos 4 estudos de caso selecionados (quatro utilizadores finais com PEA: U1, U2, U3 e U4), foi levado em consideração o desempenho de cada utilizador e as anotações feitas durante as observações 
diretas das sessões de interação com o LEMA. Estas sessões foram realizadas numa escola de referência de Portugal para alunos com PEA. Os resultados, apresentados a seguir, devem ser analisados considerando os dados fornecidos nos quadros 1 e 2.

\subsection{Desempenho do aluno U1}

O aluno U1 realizou as seguintes atividades: AT3, AT5, AT1, AT14, AT29, AT23, AT25, AT26, AT15, AT17 e AT19. Este aluno revelou ter um raciocínio geométrico estruturado em 4 atividades, um raciocínio geométrico parcialmente estruturado em 5 atividades e um raciocínio geométrico nulo em 2 atividades.

Relativamente às atividades em que o aluno U1 revelou ter um raciocínio geométrico estruturado, ele conseguiu encontrar o perímetro de uma determinada região, mostrando ter uma compreensão total do conceito de perímetro, o que significa que associa ao perímetro a medida de um objeto unidimensional mergulhado num ambiente bidimensional. Mas ele não conseguiu preencher o campo disponível para justificar a sua resposta, revelando ter um raciocínio matemático estruturado com argumento nulo (AT29). Ele foi capaz de selecionar os quadrados apropriados numa quadricula para completar uma figura usando a simetria axial (AT15).

$\mathrm{Na}$ atividade AT17, foi solicitado a identificação, entre três possibilidades da seguinte situação: dobrando uma folha duas vezes ao meio e fazendo um furo na folha dobrada, qual seria a configuração obtida quando a folha fosse desdobrada. Nesta atividade, o utilizador poderia, se assim o desejasse, assistir a um vídeo no qual ele poderia visualizar o processo de dobramento de papel descrito e a execução do buraco nele. Salienta-se o fato de esta atividade ser, sem dúvida, uma atividade de grande nível de dificuldade. O aluno U1 selecionou a resposta correta na primeira tentativa de resolução, revelando um sentido espacial total, desencadeando uma representação mental 2D-3D-2D precisa do processo de desdobramento, ainda que tenha expressado o seu raciocínio de forma imprecisa: "Eu pensei que furou um dos cantos, foi um palpite" (Registo das anotações da sessão de teste e avaliação inicial do protótipo do aluno U1, ano 2015 in SANTOS, 2018).

Na atividade AT19, foi solicitada a seleção, entre três possibilidades dadas, de qual das figuras corresponderia à figura ausente obtida por reflexão. O aluno U1 resolveu estas atividades na sua primeira tentativa, revelando um raciocínio matemático estruturado.

O aluno U1 mostrou ter dificuldade em isolar e identificar todas as figuras geométricas presentes na figura ilustrada (AT3), revelando ter dificuldades na percepção da figura-fundo. Ele também mostrou ter uma constância perceptual parcial (AT5) e, na atividade AT25, na qual 
foi solicitado a identificação de figuras com área maior/menor/igual, sendo estas figuras desenhadas em quadrículas, U1 deu resposta na sua segunda tentativa e foi capaz de justificar a sua resposta: "Eu pensei como está o círculo, a área do quadrado é maior" (Registo das anotações da sessão de teste e avaliação inicial do protótipo do aluno U1, ano 2015 in SANTOS, 2018).

De acordo com os critérios estabelecidos, o aluno U1 mostrou ter um raciocínio matemático parcialmente estruturado com argumento total nesta atividade. Na atividade AT14, este aluno teve dificuldades em diferenciar polígonos de poliedros. Na AT23, U1 só conseguiu dar a resposta correta, na terceira tentativa de resolução da atividade, depois de ter visualizado um vídeo com um feedback tutorial. De acordo com os critérios declarados, U1 mostrou nessas atividades ter um raciocínio geométrico parcialmente estruturado.

Em relação à AT26, o aluno U1 não visualizou corretamente a área triangular em consideração, definida à custa de uma diagonal de um retângulo e que por consequência a sua área correspondia à metade da área desse retângulo. Importa referir que este aluno pediu para realizar as 11 atividades selecionadas uma segunda vez e, ao interagir de novo com a AT26 confirmou-se que o aluno sabia como calcular a área de um retângulo, visto que, desta vez, a área sombreada correspondia à área de um retângulo. Porém, não conseguiu visualizar os dois triângulos definidos pelas diagonais do retângulo, mostrando ter dificuldades em calcular uma área triangular imersa numa retangular. Assim, nesta atividade, este aluno revelou ter um raciocínio matemático nulo devido principalmente à sua discriminação visual nula. $\mathrm{O}$ aluno U1 não conseguiu visualizar o cubo (entre três possibilidades) correspondente à planificação fornecida com formas geométricas em cada face, revelando que ele não foi capaz de fazer a manipulação mental de imagens e construção de relações entre imagens (AT1).

Com base nos resultados descritos acima, verificamos que o aluno U1 melhorou o seu desempenho no nível do raciocínio geométrico ao interagir com o ambiente digital LEMA, quando comparado ao estudo preliminar realizado (SANTOS; BREDA; ALMEIDA, 2015), 2015). No estudo preliminar, este estudante reconheceu figuras geométricas planares, mas não compreendeu as propriedades da reflexão. Ele mostrou dificuldade com o processo de visualização 2D-3D-2D, em fazer conjecturas e em justificar as suas afirmações, indicando um raciocínio geométrico fracamente estruturado. O seu discurso matemático também era bastante desestruturado, tal como podemos observar pelas produções deste aluno durante a sessão no estudo preliminar: "Área? O que é isso? Sou muito esquecido..." (Registo das anotações da sessão de teste e avaliação inicial do protótipo do aluno U1, ano 2015 in SANTOS, 2018), depois de perceber a explicação, diz que "a área do quadrado é 4. Há vejo também meio 
circulo..." (Registo das anotações da sessão de teste e avaliação inicial do protótipo do aluno U1, ano 2015 in SANTOS, 2018)“já estou a ficar esquecido..." "a área do circulo também é 4" (Registo das anotações da sessão de teste e avaliação inicial do protótipo do aluno U1, ano 2015 in SANTOS, 2018). (Nota o círculo estava circunscrito ao quadrado). Resumindo, U1 melhorou as suas capacidades de visualização 2D-3D-2D, descreveu partes e propriedades das figuras informalmente, embora de uma forma incompleta.

\subsection{Desempenho do aluno U2}

Averiguando o desempenho do aluno U2 ao nível da capacidade de raciocínio matemático/argumento intrínseco às 11 atividades propostas (AT4, AT14, AT31, AT8, AT12, AT5, AT25, AT28, AT18, AT21 e AT2) constatamos que, após a sua interação com o LEMA, este aluno revelou ter um raciocínio geométrico estruturado em 6 atividades, um raciocínio geométrico parcialmente estruturado em 3 atividades e um raciocínio geométrico nulo em 2 atividades.

No que concerne às atividades realizadas com raciocínio geométrico estruturado, observamos que o aluno foi capaz de identificar todas as formas geométricas presentes na figura fornecida (AT4), visualizar que seria possível construir um sólido geométrico com 10 palhinhas, sendo, no entanto, incapaz de fornecer uma justificação (AT12) para esse fato. Este aluno foi capaz de identificar figuras com área maior/menor/igual, sendo estas figuras traçadas numa grelha de quadrículas, se mostrando capaz de justificar completamente a sua resposta (AT25): "Porque o retângulo é maior que o círculo" (Registo das anotações da sessão de teste e avaliação inicial do protótipo do aluno U2, ano 2015 in SANTOS, 2018). Também conseguiu identificar facilmente a isometria (translação) apresentada no vídeo disponibilizado (AT18), identificar, entre 3 possibilidades dadas, a figura obtida pela composição de duas reflexões em eixos paralelos (AT20). Selecionou a resposta correta, entre 2 possibilidades dadas (sim ou não) relacionada com uma contagem (não trivial) de regiões triangulares de uma área sombreada determinada num hexágono (AT28), tendo, contudo, apresentado uma justificação incompleta: "Porque não dá 11" (Registo das anotações da sessão de teste e avaliação inicial do protótipo do aluno U2, ano 2015 in SANTOS, 2018).

$\mathrm{O}$ aluno U2 teve dificuldades em selecionar figuras correspondentes a polígonos. $\mathrm{Na}$ primeira tentativa de resolução da atividade AT14, U2 apenas selecionou polígonos regulares e, na segunda e terceira tentativa, selecionou polígonos convexos não regulares, esquecendo por completo os côncavos. Analisando o desempenho de U2 em AT31, na qual foi pedido para 
calcular, justificando, o perímetro de uma região hexagonal coberta duas vezes com a respectiva justificação, ele chegou à resposta certa na sua terceira tentativa e a justificação que apresentada foi completa: "9000 $6=54$ 000; $54000 \times 2=108000 ; 108000 \mathrm{~cm}=1080 \mathrm{~m}$ " (Registo das anotações da sessão de teste e avaliação inicial do protótipo do aluno U2, ano 2015 in SANTOS, 2018).

Consultando as notas de observação, pudemos constatar que nesta atividade as dificuldades dele estavam associadas à conversão das unidades de medida. Em relação à atividade AT21, na qual foi solicitada a seleção de todos os pares, entre um conjunto de 4 pares de figuras, os que representam pares de figuras congruentes, o aluno U2 revelou dificuldades em visualizar todos os pares e em dar uma justificação para as suas escolhas. De acordo com os critérios estabelecidos, este aluno mostrou ter um raciocínio geométrico parcialmente estruturado neste grupo de atividades.

Em relação às atividades em que o aluno U2 revelou ter um raciocínio geométrico nulo, tal aconteceu por ele não ter selecionado todas as planificações corretas do cubo ilustrado, entre três possibilidades (AT8). Foi capaz de selecionar corretamente uma delas, mas a outra foi selecionada por tentativa e erro (registado nas nossas anotações de observação e pela verbalização do aluno "O quê? Agora vai isto" (Registo das anotações da sessão de teste e avaliação inicial do protótipo do aluno U2, ano 2015 in SANTOS, 2018).

$\mathrm{Na}$ atividade AT5, a existência de vários retângulos cujos lados são parte dos lados de outros retângulos constituiu uma tarefa com grau de dificuldade elevado. O aluno U2 só conseguiu selecionar a resposta correta na terceira tentativa pela estratégia de tentativa e erro. Assim, este aluno mostrou, nesta atividade, ter um raciocínio matemático nulo devido principalmente à sua subcapacidade de constância perceptual.

À semelhança do aluno U1, em relação ao estudo preliminar efetuado (SANTOS; BREDA; ALMEIDA, 2015), o aluno U2 melhorou o seu nível da raciocínio geométrico ao interagir com o LEMA, uma vez que, no estudo preliminar, esse aluno mostrou dificuldades no processo de percepção 2D-3D-2D, não foi capaz de fazer conjecturas, nem de justificar as declarações, sendo que a sua comunicação matemática foi consistente com o seu desempenho: (por ex.:"A área do quadrado é 4. Do retângulo é 2. Do círculo, não dá!" "não estou a perceber" "Ficou com pontos, não estava á estava à espera disso" (Registo das anotações da sessão de teste e avaliação inicial do protótipo do aluno U2, ano 2015 in SANTOS, 2018). Ao interagir com o LEMA, este aluno melhorou as suas capacidades de visualização 2D-3D-2D e foi já capaz de justificar algumas das declarações de forma completa, tal como mencionado anteriormente. 


\subsection{Desempenho U3}

Analisando agora o desempenho do aluno U3 ao nível da capacidade de raciocínio matemático e de argumentação no universo das 11 atividades que lhe foram propostas (AT11, AT13, AT27, AT28, AT8, AT31, AT23, AT6, AT16, AT22 e AT20) verifica-se que o aluno U3 demonstrou ter um raciocínio geométrico estruturado em 5 atividades, pois ele foi capaz de selecionar a opção correta, entre as 6 possibilidades dadas, identificando ao sólido cuja planificação é formada por exatamente 5 dos 9 polígonos fornecidos (AT11), indicar o número de palhinhas necessárias para construir um prisma cuja base é um pentágono, dando uma explicação completa do seu raciocínio (AT13): “A figura representa a base de um prisma pentagonal e esse sólido tem 15 arestas e cada palhinha representa as arestas" (Registo das anotações da sessão de teste e avaliação inicial do protótipo do aluno U3, ano 2015 in SANTOS, 2018), encontrar o perímetro da região dada, fornecendo uma argumentação completa: "São necessários 1080 de rede porque a vedação do terreno corresponde ao seu perímetro e como deu 2 voltas completas com a rede percorreu 1080m" (Registo das anotações da sessão de teste e avaliação inicial do protótipo do aluno U3, ano 2015 in SANTOS, 2018), exibindo todos os cálculos associados (AT31), fazer uma contagem (não trivial) das regiões triangulares da área sombreada determinada num hexágono, justificando a sua resposta: "Porque a região sombreada corresponde a 12 triângulos e não a 11" (Registo das anotações da sessão de teste e avaliação inicial do protótipo do aluno U3, ano 2015 in SANTOS, 2018) na AT28, e, conseguiu resolver um problema envolvendo a composição de reflexões em eixos paralelos, mostrando uma compreensão total dos conceitos e propriedades da reflexão envolvidas (AT20).

O aluno U3 demonstrou ter um raciocínio geométrico parcialmente estruturado em 4 atividades, nomeadamente em encontrar a área das regiões triangulares definidas pelas diagonais de um papagaio. Este aluno deu a resposta correta na sua terceira tentativa, devido à sua ineficiente constância perceptiva e discriminação visual (AT27). Na atividade AT23, o aluno U3 só foi capaz de dar a resposta correta após a visualização do feedback tutorial fornecido, no qual a solução com todos os passos a ela conducentes foram dados.

Ele também teve dificuldades em indicar, com justificação, o número de regiões nas quais a folha de papel seria dividida após o processo de desdobramento. Após a visualização de um vídeo, U3 deu a resposta correta na terceira tentativa com uma explicação completa de seu pensamento, revelando um raciocínio geométrico parcialmente estruturado com argumento total na atividade AT16: "Porque como a folha foi dobrada 3 vezes e em cada vez no fim ficará 
dividida em 8 partes" (Registo das anotações da sessão de teste e avaliação inicial do protótipo do aluno U3, ano 2015 in SANTOS, 2018).

Além disso, este aluno não foi capaz de ver que a figura dada tinha os dois tipos de simetria na atividade AT22. U3, nas duas primeiras tentativas, não deu a resposta correta e apenas na terceira tentativa selecionou corretamente a opção correspondente a ambas simetrias, apresentando uma explicação completa da sua resposta (AT22) "Porque a figura tem ambas as simetrias e como cada menino mencionou uma simetria então os dois estão certos" (Registo das anotações da sessão de teste e avaliação inicial do protótipo do aluno U3, ano 2015 in SANTOS, 2018).

$\mathrm{Na}$ atividade AT8, este aluno revelou ter um raciocínio matemático nulo. Embora tenha sido capaz de selecionar corretamente uma das planificações do cubo ilustrado, na primeira tentativa, a outra planificação foi selecionada pela estratégia de tentativa e erro (conforme registrado nas nossas anotações de observação) na terceira tentativa de resolução desta atividade. Em relação à atividade AT6, o U3 revelou dificuldades no processo de visualização 2D envolvido, não sendo capaz de identificar todos os quadrados presentes na figura fornecida. Nas suas três tentativas, ele não obteve a resposta correta. Consequentemente, ele mostrou ter um raciocínio geométrico nulo com argumento nulo nestas atividades.

O aluno U3 ao interagir com o LEMA para além de ser capaz de reconhecer e raciocinar acerca das figuras geométricas, foi capaz de identificar e descrever as propriedades das figuras geométricas através de uma análise informal das relações entre partes de uma figura e de, em algumas atividades, espelhar a argumentação usando uma combinação da comunicação formal e informal.

\subsection{Desempenho U4}

Analisando agora o desempenho do aluno U4 ao nível da capacidade de raciocínio matemático implícitas nas 11 atividades propostas (AT2, AT18, AT15, AT24, AT25, AT7, AT9, AT32, AT30, AT17 e AT19) observa-se que ele revelou ter um raciocínio geométrico estruturado em 7 atividades, um raciocínio geométrico parcialmente estruturado em 2 atividades e um raciocínio geométrico nulo em 2 atividades.

O aluno da U4 mostrou ter uma percepção total da figura-fundo, sendo capaz de isolar e identificar todas as formas geométricas da figura fornecida (AT2), de identificar facilmente a isometria apresentada num vídeo, nomeadamente, a translação (AT18), completar uma figura com o eixo de simetria fornecido (AT15), identificar figuras de área igual/maior/menor área, 
sendo capaz de justificar completamente a sua resposta: "Porque quadrado é grande e o círculo é mais pequeno" (Registo das anotações da sessão de teste e avaliação inicial do protótipo do aluno U4, ano 2015 in SANTOS, 2018) ao realizar a AT25.

Também o aluno U4 foi capaz de visualizar a bola como um objeto representativo de uma esfera (AT7), identificar facilmente o sólido geométrico e a sua respectiva planificação (AT9) e determinar o perímetro de um cartão retangular, dando uma justificação completa da sua resposta: "Somei os dois números duas vezes" (Registo das anotações da sessão de teste e avaliação inicial do protótipo do aluno U4, ano 2015 in SANTOS, 2018), revelando uma compreensão total do conceito geométrico de perímetro (AT30). O aluno resolveu estas atividades na primeira tentativa de resolução, revelando ter um raciocínio geométrico estruturado com total argumentação.

Quanto à atividade AT24, foi solicitada a identificação da área de uma figura tomando um triângulo como a unidade de medida. $\mathrm{O}$ aluno $\mathrm{U} 4$, na primeira tentativa de resolução, não deu a resposta correta, verbalizando “isto é difícil. É muito complicado!" (Registo das anotações da sessão de teste e avaliação inicial do protótipo do aluno U4, ano 2015 in SANTOS, 2018). Após uma explicação oral da professora sobre os conceitos envolvidos, conseguiu dar a resposta correta na segunda tentativa, revelando um raciocínio matemático parcialmente estruturado nesta atividade. Assim como na atividade AT17, já descrita, é, sem dúvida, uma atividade de elevada dificuldade (é necessária a construção mental dos processos de desdobramento é necessária). O aluno U4 selecionou a resposta correta na sua segunda tentativa dando uma explicação inexata: "Vendo o vídeo algumas vezes" (Registo das anotações da sessão de teste e avaliação inicial do protótipo do aluno U4, ano 2015 in SANTOS, 2018), demonstrando dificuldades na construção mental do processo de desdobramento da folha e da folha quando desdobrada. Assim, este aluno revelou ter um raciocínio matemático parcialmente estruturado com argumento nulo.

O aluno U4 revelou ter raciocínio geométrico nulo nas atividades AT32 e AT19 porque, em primeiro lugar, ele não foi capaz de determinar o perímetro de um campo de futebol dividido em três quadrados, dois dos quais geometricamente iguais. Em segundo, ele resolveu na primeira tentativa, mas, observando as nossas anotações de observações, sabemos que essa resposta foi dada por acaso.

Comparando o desempenho do aluno U4 no estudo preliminar (SANTOS; BREDA; ALMEIDA, 2015) e ao interagir com o LEMA, verifica-se que este aluno melhorou o seu nível de raciocínio geométrico ao interagir com o ambiente digital proposto. No estudo preliminar, este aluno não tinha adquirido ainda o conceito de área, utilizou o pensamento visual de forma 
ineficiente, falhando no processo de percepção 2D-3D-2D. Constatou-se ainda que a comunicação matemática deste aluno era desestruturada, como observamos através das suas declarações: “Área ... respondo daqui a 6 segundos!... isto é como se estivesse na aula e não me está a agradar... Não me apetece... estou a pensar. São 4 figuras iguais..." "Não sei o quê mas vai acontecer alguma coisa!" (Registo das anotações da sessão de teste e avaliação inicial do protótipo do aluno U4, ano 2015 in SANTOS, 2018). Ao interagir com LEMA, este aluno melhorou as suas capacidades de visualização 2D-3D-2D, pois já era capaz de justificar algumas das suas declarações de forma completa, tal como vimos antes, e agora reconheceu e raciocinou acerca das figuras geométricas planas.

Considerando as opiniões dos professores acompanhantes, todos os entrevistados relataram o LEMA como sendo um ambiente digital intuitivo, com uma navegação simples e eficiente, amigável e apropriado às crianças/jovens com PEA, sublinhando a importância do LEMA incorporar enunciados simples, claros e objetivos acoplados com imagens ilustrativas e apresentar uma ambiente gráfico bem elaborado.

Da mesma forma, a totalidade dos entrevistados se referiu à adequação dos feedbacks de reforço, contudo um entrevistado destacou o fato de alguns feedbacks serem um pouco infantilizados e outro mencionou que alguns feedbacks podiam ser mais objetivos. Quanto à adequação dos feedbacks tutoriais, importa sublinhar um comentário referido por um dos docentes que indicou que os feedbacks tutoriais não são os mais adequados, sugerindo que estes, em vez de dar as respostas, fossem antes pistas para a resolução das atividades, o que ajudaria no desenvolvimento do raciocínio.

No que diz respeito à utilização do LEMA por parte dos alunos, a totalidade das professoras entrevistadas mencionou que a quantidade de informação em cada tela e o ambiente gráfico do LEMA são adequados aos utilizadores com PEA, bem como LEMA atende aos conhecimentos, aptidões linguísticas e compreensão dos utilizadores. Questionadas acerca das telas que despertam maior atenção, a totalidade dos entrevistados referiu as telas que integram a seleção de itens e aquelas que incorporam a observação de vídeos.

Em relação à questão sobre o interesse, utilidade e importância que o LEMA desperta no desenvolvimento do raciocínio matemático, as entrevistadas referiram que o LEMA reforça a interiorização e a consolidação das aprendizagens. $O$ fato de ser um ambiente audiovisual motiva e facilita a aprendizagem, tendo ainda sido realçado o fato de as atividades implementadas no LEMA e o feedback tutorial contribuírem para o desenvolvimento do raciocínio matemático.

Apesar da totalidade das professoras referir que as atividades incorporadas ao LEMA 
são estruturadas e adaptadas aos estilos de aprendizagem de crianças/jovens com PEA, todos referiam que considerando, o universo das PEA, o LEMA só se adequa a alguns perfis de utilizadores, sugerindo que, para abranger um maior número de utilizadores com PEA, em algumas atividades teria que haver uma maior estruturação, o desmembramento de uma atividade em subtarefas e a diminuição de grau de dificuldade. Igualmente indicaram que as estratégias utilizadas no LEMA para a promoção do raciocínio matemático são eficazes.

Importa referir duas sugestões de outras estratégias referidas por uma das professoras entrevistadas: que as instruções das atividades fossem dadas mais passo a passo e que houvesse uma explicação dos conceitos ainda mais pormenorizada.

Todas as professoras sublinharam a importância do LEMA para o desenvolvimento do raciocínio matemático no contexto da intervenção da PEA. As atividades implementadas no LEMA promovem o desenvolvimento do raciocínio matemático, bem como a atenção, concentração e autonomia nos alunos com PEA, caraterísticas que normalmente são deficitárias nestes alunos. Outras vantagens mencionadas pelas professoras referem-se ao LEMA enquanto ambiente organizado e estruturado, com cores adequadas, o que vai ao encontro das caraterísticas definidas na especificação deste protótipo.

Questionadas sobre a pertinência da utilização deste ambiente digital de aprendizagem em sala de aula, a totalidade das entrevistadas referiu ser uma mais-valia para motivar a aprendizagem da Matemática. A totalidade das professoras referiu que gostaria de utilizar o LEMA no futuro, apontando as seguintes razões: a falta de material adequado às PEA para a promoção do desenvolvimento do raciocínio matemático e o fato deste ambiente digital de aprendizagem ser uma ferramenta motivadora, que poderá levar os utilizadores com PEA a desenvolverem o raciocínio e consolidem as suas aprendizagens.

\section{Conclusão}

A avaliação da capacidade de raciocínio matemático levou-nos a concluir que os alunos participantes apresentaram um raciocínio matemático parcialmente estruturado ou estruturado na maioria das atividades propostas. Durante a execução das atividades, os alunos U1, U2 e U4 revelaram que reconhecem e raciocinam acerca das figuras geométricas planas e outras configurações geométricas de acordo com a sua aparência como um todo visual, descrevendo as partes e as propriedades das figuras informalmente, variando a precisão dessa descrição. Apresentam ainda dificuldades em estabelecer relações entre as propriedades das figuras. Em relação ao aluno com melhor desempenho, o aluno U3, para além de ser capaz de reconhecer e 
raciocinar acerca das figuras geométricas, foi capaz de identificar e descrever as propriedades das figuras geométricas através de uma análise informal das relações entre partes de uma figura e de, em algumas atividades, espelhar a argumentação usando uma combinação da comunicação formal e informal.

Nesse contexto, constatamos que os alunos que participaram deste estudo melhoraram o desempenho ao nível do raciocínio geométrico ao interagir com o ambiente digital LEMA, quando comparado com os resultados obtidos no estudo preliminar realizado (SANTOS; BREDA; ALMEIDA, 2015) mostrando ter um raciocínio geométrico parcialmente estruturado ou estruturado a partir da interação com o LEMA.

De acordo com a opinião das professoras que acompanharam os alunos durante as sessões de teste e avaliação inicial, o protótipo desenvolvido é intuitivo, de fácil utilização, de navegação simples e amigável às crianças com PEA, atendendo às suas necessidades, conhecimentos e aptidões linguísticas. Os dados recolhidos nas entrevistas também são indicadores de que o protótipo incentiva o desenvolvimento do raciocínio, na medida em que reforça a consolidação das aprendizagens matemáticas.

Como o LEMA foi orientado com base nos princípios de desenho universal para a aprendizagem que incorpora o design flexível de cenários com opções personalizáveis, proporciona que todos os alunos tenham acesso ao progresso das suas aprendizagens individuais. Neste sentido, apesar do LEMA ter sido concebido e desenvolvido especificamente para atender às especificidades das crianças com PEA, também pode ser utilizado por qualquer criança/jovem com ou sem necessidades educativas especiais, dado que apresenta níveis de flexibilidade e adaptação das atividades incorporadas tendo em conta as especificidades dos utilizadores.

A fase de avaliação inicial do protótipo foi muito importante para melhorar a interface do utilizador, redesenhar algumas atividades incorporadas no protótipo e para corrigir todas as fragilidades detectadas ao longo das sessões com os quatro utilizadores finais, a fim de aperfeiçoarmos a versão final. Os dados recolhidos forneceram importantes indicadores para o melhoramento do protótipo e permitiram avançar com as primeiras reformulações do mesmo, para que efetivamente possa promover o desenvolvimento de capacidades matemáticas, atendendo à heterogeneidade que estas crianças e jovens tipicamente manifestam. Neste sentido, com base nos dados analisados, os reajustamentos do LEMA devem ser conduzidos, a fim de atender aos seguintes critérios: (1) indicações ao longo da realização das atividades, de forma que o utilizador tenha noção das atividades que já realizou, das atividades que faltam concluir; (2) repartição dos problemas matemáticos mais complexos em tarefas mais simples; 
(3) incorporação de feedback tutorial fornecido através de pistas passo a passo; (4) incorporação de feedbacks de reforço mais específicos; (5) diminuição do grau de dificuldade de algumas atividades atendendo à diversidade de perfis de funcionalidade dos utilizadores com tal perturbação; (6) incorporação de áudio nos enunciados; (7) explicação de conceitos matemáticos inerentes a cada tarefa matemática; (8) incorporação de mais atividades que promovam o desenvolvimento da orientação e visualização espacial de acordo com as três famílias de tarefas apresentadas por Gonzato, Fernández Blanco e Godino (2011).

O próximo passo neste projeto de investigação será a implementação dos reajustes acima mencionados, seguidos de posterior validação com utilizadores com PEA.

\section{Agradecimentos}

Este estudo foi suportado pela Fundação Portuguesa para a Ciência e Tecnologia (FCT) através do Centro de Investigação e Desenvolvimento em Matemática e Aplicações - CIDMA, no âmbito do projeto UID/MAT/04106/2020. M. I. Santos também reconhece o apoio da FCT através da bolsa de doutoramento SFRH / BD / 108154/2015.

\section{Referências}

ABRANTES, P.; SERRAZINA, L.; OLIVEIRA, I. A Matemática na Educação Básica. Lisboa: ME/DEB, 1999.

AMERICAN PSYCHIATRIC ASSOCIATION. Diagnostic and Statistical Manual of Mental Disorders. [s.l.] American Psychiatric Association, 2013.

BAE, Y. S.; CHIANG, H.-M.; HICKSON, L. Mathematical Word Problem Solving Ability of Children with Autism Spectrum Disorder and their Typically Developing Peers. Journal of Autism and Developmental Disorders, New York, v. 45, n. 7, p. 2200-2208, jul. 2015.

BARON-COHEN, S. Autism, maths, and sex: the special triangle. The Lancet Psychiatry, New York, v. 2, n. 9, p. 790-791, 2015.

BIVAR, A. et al. Programa e Metas Curriculares. Matemática. 1. ed. Lisboa, Portugal: Ministério da Educação e Ciência, 2013.

BURTON, C. E. et al. Video self-modeling on an iPad to teach functional math skills to adolescents with autism and intellectual disability. Focus on Autism and Other Developmental Disabilities, United States, v. 28, n. 2, p. 67-77, 2013.

CHABANI, E.; HOMMEL, B. Visuospatial processing in children with autism: No evidence for (training-resistant) abnormalities. Journal of Autism and Developmental Disorders, New York, v. 44, n. 9 , p. $2230-2243,2014$.

CHANG, K.-E. et al. Using mobile devices to enhance the interactive learning for spatial geometry. 
Interactive Learning Environments, London, v. 23, n. 1, p. 1-19, ago. 2014.

CHIANG, H.; LIN, Y. Mathematical ability of students with Asperger syndrome and high-functioning autism A review of literature. Autism, United States, v. 11, n. 6, p. 547-56, nov. 2007.

DELMOLINO, L. et al. The use of instructive feedback for teaching language skills to children with autism. Research in Autism Spectrum Disorders, Netherlands, v. 7, n. 6, p. 648-661, jun. 2013.

DEPARTMENT FOR EDUCATION AND SKILLS. The daily mathematics lesson: Guidance to support pupils with autistic spectrum disorders. Manchester, United Kingdom: Department for Education and Skills, 2001.

ENGLISH, L. D. Revealing and capitalising on young children's madothematical potential. ZDM Mathematics Education, Berlim, v. 48, n. 7, p. 1079-1087, 2016.

FREIMAN, V.; POLOTSKAIA, E.; SAVARD, A. Using a computer-based learning task to promote work on mathematical relationships in the context of word problems in early grades. ZDM Mathematics Education, Berlim, v. 49, n. 6, p. 835-849, 2017.

GEVARTER, C. et al. Mathematics Interventions for Individuals with Autism Spectrum Disorder: A Systematic Review. Review Journal of Autism and Developmental Disorders, New York, v. 3, n. 3, p. 224-238, 2016.

GONZATO, M.; FERNÁNDEZ BLANCO, M. T.; D. GODINO, J. Tareas para el desarrollo de habilidades de visualización y orientación espacial. Números. Revista de Didáctica de las Matemáticas, Espanha, v. 77, p. 99-117, 2011.

IUCULANO, T. et al. Brain organization underlying superior mathematical abilities in children with autism. Biological psychiatry, Netherlands, v. 75, n. 3, p. 223-30, fev. 2014.

JONES, C. R. G. et al. Reading and arithmetic in adolescents with autism spectrum disorders: Peaks and dips in attainment. Neuropsychology, United States, v. 23, n. 6, p. 718-728, 2009.

KING, S. A.; LEMONS, C. J.; DAVIDSON, K. A. Math Interventions for Students With Autism Spectrum Disorder: A Best-Evidence Synthesis. Exceptional Children, United States, v. 82, n. 4, p. 443-462, jul. 2016.

MATA-PEREIRA, J.; PONTE, J. P. DA. Promover o Raciocínio Matemático dos Alunos: uma investigação baseada em design. Bolema: Boletim de Educação Matemática, Rio Claro, v. 32, n. 62, p. 781-801, dez. 2018.

ME-DEB. Currículo nacional do ensino básico. Competências essenciais. Lisboa: Ministério da Educação, 2001.

NCTM - NATIONAL COUNCIL OF TEACHERS OF MATHEMATICS. Executive Summary: Principles and Standards for School Mathematics. In: Principles and Standards for School Mathematics. Reston, VA: National Council of Teachers of Mathematics, 2000. p. 1-6.

NCTM - NATIONAL COUNCIL OF TEACHERS OF MATHEMATICS. Princípios e normas para a Matemática escolar. Lisboa: APM, 2007.

OECD. PISA 2015 Assessment and Analytical Framework: Science, Reading, Mathematic, Financial Literacy and Collaborative Problem Solving. Revised Edition. Paris: PISA, OECD Publishing, 2017a

OECD. PISA 2015 Mathematics Framework. Revised Edition. Paris: PISA, OECD Publishing, 2017b 
SANTOS, M. I. As tecnologias digitais no apoio ao desenvolvimento do Raciocínio Matemático em alunos com Perturbação do Espetro do Autismo. Aveiro: Tese de Doutoramento em Multimédia em Educação da Universidade de Aveiro, 2018.

SANTOS, M. I.; BREDA, A.; ALMEIDA, A. M. Brief Report: Preliminary Proposal of a Conceptual Model of a Digital Environment for Developing Mathematical Reasoning in Students with Autism Spectrum Disorders. Journal of Autism and Developmental Disorders, New York, v. 45, n. 8, p. 2633 2640, ago. 2015.

SANTOS, M. I.; BREDA, A.; ALMEIDA, A. M. Design approach of mathematics learning activities in a digital environment for children with autism spectrum disorders. Educational Technology Research and Development, Boston, v. 65, n. 5, p. 1305-1323, out. 2017.

SHANE, H. C. et al. Applying technology to visually support language and communication in individuals with autism spectrum disorders. Journal of autism and developmental disorders, New York, v. 42, n. 6, p. 1228-35, jun. 2012.

SU, H. F. H. "ANGIE". Using an Exploratory Approach to Help Children with Autism Learn Mathematics. Creative Education, United States, v. 01, n. 03, p. 149-153, 2010.

SU, H.; LAI, L.; RIVERA, H. Effective Mathematics Strategies for Pre-School Children with Autism. Australian Primary Mathematics Classroom, v. 17, n. 2, p. 25-30, 2012.

VAN DOOREN, W.; INGLIS, M. Inhibitory control in mathematical thinking, learning and problem solving: a survey. ZDM - Mathematics Education, Berlim, v. 47, n. 5, p. 713-721, 2015.

WEI, X. et al. Reading and math achievement profiles and longitudinal growth trajectories of children with an autism spectrum disorder. Autism, United States, v. 19, n. 2, p. 200-210, fev. 2015.

YAMADA, T. et al. Functional Alterations in Neural Substrates of Geometric Reasoning in Adults with High-Functioning Autism. PLoS ONE, Osaka - Japão, v. 7, n. 8, p. e43220, ago. 2012.

Submetido em 27 de Setembro de 2019. Aprovado em 16 de Fevereiro de 2020. 